\title{
Efficacy of eculizumab in refractory life-threatening warm autoimmune hemolytic anemia associated with chronic myelomonocytic leukemia.
}

Anne-Cécile Gauchy ${ }^{1}$, Maxime Hentzien ${ }^{1}$, Alain Wynckel $^{2}$, Victoire De Marcellus ${ }^{1}$, Cyrielle Rodier $^{1}$, Alain Delmer ${ }^{1}$, and Anne Quinquenel ${ }^{1}$

${ }^{1}$ Hopital Robert Debré

${ }^{2}$ Hôpital Maison Blanche

April 28, 2020

\begin{abstract}
We report a case of refractory life-threatening warm autoimmune hemolytic anemia (wAIHA) associated with chronic myelomonocytic leukemia (CMML) for which all conventional treatments had failed. The use of eculizumab allowed controlling hemolysis and resulted in transfusion independence. These data support the use of eculizumab in refractory wAIHA.
\end{abstract}

Efficacy of eculizumab in refractory life-threatening warm autoimmune hemolytic anemia associated with chronic myelomonocytic leukemia

Anne-Cécile Gauchy ${ }^{1,2}$, Maxime Hentzien ${ }^{2,3}$, Alain Wynckel $^{4}$, Victoire de Marcellus ${ }^{1,2}$, Cyrielle Rodier ${ }^{1,2}$, Alain Delmer ${ }^{1,2}$, Anne Quinquenel $^{1,2}$

1 - Service d'Hématologie Clinique Centre Hospitalier Universitaire Hôpital Robert Debré Reims France.

2 - UFR Médecine Université Reims Champagne-Ardenne Reims France.

3 - Service de Médecine Interne, Maladies Infectieuse et Immunologie Clinique Centre Hospitalier Universitaire Hôpital Robert Debré Reims France.

4 - Service de Néphrologie Centre Hospitalier Universitaire Hôpital Robert Debré Reims France.

\section{Corresponding author}

Anne Quinquenel

aquinquenel@chu-reims.fr

\section{Keywords}

Eculizumab - warm autoimmune hemolytic anemia - chronic myelomonocytic leukemia

\section{Key Clinical Message}

Eculizumab may be considered as an emergency therapeutic option in refractory life-threatening warm autoimmune hemolytic anemia especially if direct antiglobulin test is positive for both IgG and C3d and after failure of all conventional treatments.

\section{Introduction}


Chronic myelomonocytic leukemia (CMML) is a clonal hematopoietic stem cell disorder characterized by overlapping features of myelodysplastic syndromes and myeloproliferative neoplasms. Autoimmune and inflammatory diseases (AID) are classical complications of CMML, occurring in up to $20 \%$ of patients in monocentric retrospective studies $(1,2)$. These complications can precede the onset of CMML, be concomitant to the diagnosis or occur later during the course of the disease. CMML-associated AID are as various as systemic vasculitis, connective tissue diseases, skin diseases such as neutrophilic dermatosis, polychondritis or seronegative inflammatory arthritis (1-3). Autoimmune cytopenias (AIC) have also been reported, encompassing mainly immunologic thrombocytopenias and AIHA (2-4). Based on the optimal binding temperature of autoantibodies, AIHA can be classified into three categories, warm AIHA (wAIHA), cold AIHA and mixed forms AIHA. The few reported cases of CMML-associated AIHA were wAIHA and their rarity precludes definite therapeutic recommendations to be drawn. As with AIHA observed in other settings, frontline treatment is mainly based on corticosteroids but steroid dependence is frequent. Efficacy of subsequent treatments has not been reported, conversely to CMML-associated ITP where the second line treatments used in primary ITP have proved efficacy. Eculizumab (Soliris@, Alexion Pharmaceuticals) is a humanized monoclonal antibody that binds with high affinity to the C5 complement protein and blocks its cleavage in two components: C5a and C5b (5). It is approved for the treatment of paroxysmal nocturnal hemoglobinuria $(\mathrm{PNH})$ and atypical hemolytic uremic syndrome. Because of the pivotal role of complement in cold agglutinin disease (CAD), eculizumab has also been evaluated in this disease and provided responses in rituximab refractory cases. Eculizumab has also been used in anecdotal cases of wAHAI but none of them were associated with CMML. We report herein the efficacy of eculizumab in a case of life-threatening refractory wAIHA associated with CMML.

\section{Case history}

A 70-year-old woman, followed at our institution for CMML associated with wAIHA, was admitted at hospital for dyspnea and malaise in March 2017. At the time of CMML diagnosis 15 years earlier, in 2002, she presented with splenomegaly, oral lichen planus and IgG-mediated wAIHA. At that time, bone marrow examination did not show excess of blasts and karyotype was normal. CMML did not require any treatment by itself but treatment for symptomatic anemia was initiated. AIHA responded initially to prednisone (1 $\mathrm{mg} / \mathrm{kg} /$ day) but relapses were frequent during tapering and the patient experienced many steroids complications. Mycophenolic acid failed to control the hemoglobin level and the patient required repeated packed red blood cells (PRBC) transfusions. A splenectomy was performed in 2007, leading to a prolonged control of AIHA. The hemoglobin level remained stable between 100 and $110 \mathrm{~g} / \mathrm{L}$ until 2015, although haptoglobin level was repeatedly below the normal range, and then it gradually decreased. In the meantime, hydroxyurea had been initiated in June 2008, because of increasing leukocytosis. Treatment with recombinant erythropoietin was initiated in November 2016, because anemia was thought to be more related to bone marrow failure than hemolysis at that time. Erythropoietin was ineffective as well as prednisone which was resumed at the beginning of 2017. The patient received several RBCP transfusions, especially after hip prosthesis for intercurrent femoral neck fracture, but their efficacy was very transient. Thrombocytopenia then developed for the first time in the history of the disease. At admission in March 2017, hemoglobin level was $63 \mathrm{~g} / \mathrm{L}$ with reticulocytes count $227 \mathrm{G} / \mathrm{L}$, platelet count was $61 \mathrm{G} / \mathrm{L}$ and white blood cells were $23 \mathrm{G} / \mathrm{L}$ with $22 \%$ monocytes and 15\% myelocytes and metamyelocytes. There were both elevation of LDH level (1.5 x UNV) and decreased haptoglobin level $(<0.10 \mathrm{~g} / \mathrm{L})$. Direct antiglobulin test (DAT) was positive for IgG (strong) and C3d (low). Bone marrow aspiration showed both erythroblastic and monocytic hyperplasia without blast excess, thus eliminating leukemic transformation. The megakaryocytes were normally present, suggesting that thrombocytopenia was related to peripheral destruction of platelets. Screen for cold agglutinins and a paroxysmal nocturnal hemoglobinuria (PNH) clone was negative. The diagnosis of Evans syndrome was established. The dose of prednisone was increased to $2 \mathrm{mg} / \mathrm{kg} /$ day and the patient then received, within a short period of time, daily boluses of high-dose methylprednisolone $(500 \mathrm{mg} /$ day for three days), intravenous immunoglobulins $\left(1 \mathrm{~g} / \mathrm{kg} /\right.$ day for two consecutive days) and four infusions of rituximab ( $375 \mathrm{mg} / \mathrm{m}^{2}$ weekly). All these treatments failed and several plasma exchanges were performed, due to poor anemia tolerance with a hemoglobin level of $31 \mathrm{~g} / \mathrm{L}$ at nadir. Despite all these procedures, hemoglobin level did not improve nor 
stabilize. About one month after the last infusion of rituximab, a treatment with eculizumab was started. The patient received $600 \mathrm{mg}$ of eculizumab every week for four weeks and then $900 \mathrm{mg}$ every two weeks. After starting eculizumab, hemoglobin and platelet levels gradually increased. Three months later, hemoglobin has stabilized around $10 \mathrm{~g} / \mathrm{L}$, while the platelet and LDH levels were normalized (table 1). For the first time in her medical story, the haptoglobin level went back to near normal values. The infusions of eculizumab were progressively spaced out and then definitively stopped in July 2018, after 14 months of treatment. With a follow-up of 12 additional months, no recurrence of AIHA has occurred.

\section{Discussion}

We report herein the first success to our knowledge of eculizumab for the treatment of wAIHA occurring in a patient with CMML.

Even if accurate data are lacking concerning the prevalence of inflammatory or autoimmune diseases in CMML patients, monocentric studies suggest that AID may occur in about $20 \%$ of cases $(1,2)$. The outcome of patients with CMML-associated AID remains unclear, but in most cases, they exhibit rather favorable prognostic features of the underlying myeloid disorder. In a retrospective study of 377 CMML patients, those with AID were more likely to have a lower risk prognostication based on the Mayo Clinic prognostic model (1). Results were similar in a French retrospective study (3). In a third cohort, cytogenetic abnormalities seemed less frequent in CMML patients with AID (2). Finally, in a small cohort focusing on CMML-associated ITP, all cases of immune thrombocytopenia occurred in patients with low-risk CMML, and none of them experienced CMML progression or transformation into acute myeloid leukemia during follow-up (4). Considering our patient, CMML has remained stable with a follow-up of 17 years.

Among AIC, the development of ITP seems much more frequent than AIHA. Thus, the only available data reporting efficacy of treatments in CMML-associated autoimmune cytopenias concern patients with ITP. In nearly all reported cases of CMML-associated AID, first-line treatment consisted of corticosteroids, and most relapsed patients received immunosuppressive therapies. Focusing on ITP, successful treatments with corticosteroids, rituximab, splenectomy or thrombopoietin agonists have been reported, and some authors consider that CMML-associated ITP should be treated according to current guidelines for primary ITP (4). The same rationale could apply to CMML-associated AIHA and in our patient; splenectomy led to a durable control of AIHA for about 10 years.

In AIC associated with lymphoproliferative disorders such as chronic lymphocytic leukemia, most experts believe that an optimal control of the malignant clone would represent the best option to control autoimmune manifestations. The same hypothesis could be raised in CMML-associated AID. The rationale for the use of 5-azacitidine in this setting, even in the absence of CMML progression, is based on its ability to remove or at least to lower the tumor clone. Some studies have reported efficacy of 5-azacitidine in autoimmune manifestations associated with myelodysplastic syndromes or CMML but none of them was AIHA. A prospective phase II trial, supported by French Group of Myelodysplasia (NCT02985190), is currently evaluating the efficacy and safety of 5-azacitidine in refractory autoimmune and inflammatory diseases associated with myelodysplastic syndromes, and may provide answers for clinicians. However, azacitidine cannot be considered as a salvage treatment in case of life-threatening AIC because responses are usually delayed $(6,7)$. Moreover, its initial myelotoxicity may worsen the cytopenias.

The use of eculizumab is not approved for AIHA. A prospective phase II trial reported its efficacy in patients with CAD (8). These results can be explained by the central role of terminal complement activation in cold AIHA. The use of eculizumab has already been reported in a case of wAIHA associated with Waldenstrom macroglobulinemia (9). In this case, wAIHA was also refractory to steroids, rituximab and plasmapheresis. As in our patient, DAT was positive for both IgG and C3d. After four weekly doses of eculizumab hemoglobin was stabilized around $90 \mathrm{~g} / \mathrm{L}$ and treatment was stopped. The patient remained in partial remission for four months before he died from refractory anemia relapse. The complement system plays an important role in the physiopathology of AIHA. Some patients, including our case, suffer from wAIHA with both IgG and C3d positive DAT. In these specific forms, autoantibodies, especially IgM, can activate the complement 
system in vivo, leading to the destruction of red blood cells. These IgM, which react at $37 \mathrm{deg}$, contribute to hemolysis by activating the classical pathway of the complement system. Meulenbroek et al. showed that all patients with $\mathrm{C} 3$ on their red blood cells have an IgM component that could activate complement even if no such antibody could be detected with routine techniques (10). In wAIHA with positive DAT for C3d, complement-mediated red blood cell destruction contributes to the severity of acute hemolysis, exacerbations of chronic AIHA and poor performance of red blood cell transfusions. For these patients, use of eculizumab could prevent red blood cell transfusions, which can exacerbate hemolysis, or could restore the transfusion efficiency, while waiting for the effect of the other treatments. In addition to its cost, the suspensive effect and the risk of severe bacterial infections such as meningococcal infection could represent the main limitation of eculizumab (11). In this specific population, it is of major interest to consider the safety profile of eculizumab since previous exposure to corticosteroids or other immunosuppressive agents might increase even more the risk of severe infections. We did not observe any infectious complication during our patient's treatment with eculizumab. In the prospective study on CAD, Roth et al, reported one case of treatment-related pneumonia among 13 patients treated with eculizumab (8). No meningococcal infection was observed.

In our case, a delayed effect of rituximab cannot be formally ruled out but the efficacy of this treatment is usually not observed beyond three months (12). The concomitant correction of thrombocytopenia is a further argument for rituximab late effect because of its proven efficacy in both primary and secondary ITP (13). Moreover, complement system has not been involved in autoimmune thrombocytopenia until now. Whether eculizumab should have been pursued such a long time in our patient is questionable. In the phase 2 study on CAD, eculizumab was given for 26 weeks (8). As our patient did not experience any infectious complication and because of the severity of AHAI presentation, eculizumab was maintained up to 56 weeks, but it may have been stopped earlier. One year after the end of treatment, no relapse has occurred so far. In case of AIHA relapse, a new course of rituximab or 5 -azacitidine could be considered but since these agents have an expected delayed efficacy, eculizumab should be rechallenged if needed.

\section{Conclusion}

In summary, our case report supports the use of eculizumab as an emergency therapy in refractory lifethreatening wAIHA especially if DAT is positive for both IgG and C3d, even if related to underlying hematological malignancy and after failure of conventional therapies. However, this treatment is suspensive and should only be considered as a transient salvage treatment. Optimal duration, dosage and frequency of administration are unknown.

\section{Conflict of interest}

The authors declare that there are no conflicts of interests regarding the publication of this article.

\section{Author contributions}

ACG: contributed to patient evaluation, acquired the data, analyzed and interpreted the data, wrote the manuscript, and approved the final manuscript. AW and $\mathrm{MH}$ : contributed to patient evaluation and approved the final manuscript. VDM, CR, AQ and AD: analyzed and interpreted the data, wrote and approved the final manuscript.

\section{References}

1. Zahid MF, Barraco D, Lasho TL, Finke C, Ketterling RP, Gangat N, et al. Spectrum of autoimmune diseases and systemic inflammatory syndromes in patients with chronic myelomonocytic leukemia. Leuk Lymphoma. 2017 Jun;58(6):1488-93.

2. Peker D, Padron E, Bennett JM, Zhang X, Horna P, Epling-Burnette PK, et al. A close association of autoimmune-mediated processes and autoimmune disorders with chronic myelomonocytic leukemia: observation from a single institution. Acta Haematol. 2015;133(2):249-56. 
3. Grignano E, Mekinian A, Braun T, Liozon E, Hamidou M, Decaux O, et al. Autoimmune and inflammatory diseases associated with chronic myelomonocytic leukemia: A series of 26 cases and literature review. Leuk Res. 2016 Aug;47:136-41.

4. Hadjadj J, Michel M, Chauveheid M-P, Godeau B, Papo T, Sacre K. Immune thrombocytopenia in chronic myelomonocytic leukemia. Eur J Haematol. 2014 Dec;93(6):521-6.

5. Wouters D, Zeerleder S. Complement inhibitors to treat IgM-mediated autoimmune hemolysis. Haematologica. 2015 Nov;100(11):1388-95.

6. Fraison J-B, Mekinian A, Grignano E, Kahn J-E, Arlet J-B, Decaux O, et al. Efficacy of Azacitidine in autoimmune and inflammatory disorders associated with myelodysplastic syndromes and chronic myelomonocytic leukemia. Leuk Res. 2016 Apr;43:13-7.

7. Scott LJ. Azacitidine: A Review in Myelodysplastic Syndromes and Acute Myeloid Leukaemia. Drugs. 2016 May;76(8):889-900.

8. Roth A, Bommer M, Huttmann A, Herich-Terhurne D, Kuklik N, Rekowski J, et al. Eculizumab in cold agglutinin disease (DECADE): an open-label, prospective, bicentric, nonrandomized phase 2 trial. Blood Adv. 2018 09;2(19):2543-9.

9. Ma K, Caplan S. Refractory IgG Warm Autoimmune Hemolytic Anemia Treated with Eculizumab: A Novel Application of Anticomplement Therapy. Case Rep Hematol. 2016;2016:9181698.

10. Meulenbroek EM, de Haas M, Brouwer C, Folman C, Zeerleder SS, Wouters D. Complement deposition in autoimmune hemolytic anemia is a footprint for difficult-to-detect IgM autoantibodies. Haematologica. 2015 Nov;100(11):1407-14.

11. Benamu E, Montoya JG. Infections associated with the use of eculizumab: recommendations for prevention and prophylaxis. Curr Opin Infect Dis. 2016;29(4):319-29.

12. Reynaud Q, Durieu I, Dutertre M, Ledochowski S, Durupt S, Michallet A-S, et al. Efficacy and safety of rituximab in auto-immune hemolytic anemia: A meta-analysis of 21 studies. Autoimmun Rev. 2015 Apr;14(4):304-13.

13. Zaja F, Volpetti S, Chiozzotto M, Puglisi S, Isola M, Buttignol S, et al. Long-term follow-up analysis after rituximab salvage therapy in adult patients with immune thrombocytopenia. Am J Hematol. 2012 Sep;87(9):886-9.

Table 1 - Evolution of biological parameters after eculizumab treatment

\begin{tabular}{llllll}
\hline $\begin{array}{l}\text { Delay after the } \\
\text { first injection } \\
\text { of eculizumab }\end{array}$ & $\begin{array}{l}\text { Hemoglobin } \\
(\mathrm{g} / \mathbf{L})\end{array}$ & $\begin{array}{l}\text { Platelets } \\
(\mathbf{G} / \mathbf{L})\end{array}$ & $\begin{array}{l}\text { Haptoglobin } \\
(\mathrm{g} / \mathbf{L})\end{array}$ & $\mathbf{L D H}$ (UI/L) & $\begin{array}{l}\text { Reticulocytes } \\
\text { (G/L) }\end{array}$ \\
\hline $\mathbf{1}$ month & 38 & 19 & $<0,01$ & 449 & Not available \\
2 months & 68 & 109 & $<0,01$ & 377 & Not available \\
3 months & 97 & 124 & 0,24 & 316 & Not available \\
6 months & 108 & 127 & 0,16 & 404 & Not available \\
12 months & 115 & 171 & 0,23 & 345 & 47 \\
14 months & 112 & 198 & 0,3 & 272 & 78 \\
\hline
\end{tabular}

